\title{
Simultaneous quantum dash-well emission in a chirped dash-in-well superluminescent diode with spectral bandwidth $>700 \mathrm{~nm}$
}

\author{
M. Z. M. Khan, ${ }^{1}$ M. A. Majid, ${ }^{1}$ T. K. Ng, ${ }^{1}$ D. Cha, ${ }^{2}$ and B. S. Ooi ${ }^{1, *}$ \\ ${ }^{1}$ Photonics Laboratory, Computer, Electrical, and Mathematical Sciences and Engineering (CEMSE) Division, \\ King Abdullah University of Science \& Technology (KAUST), Thuwal 23955-6900, Saudi Arabia \\ ${ }^{2}$ Advanced Nanofabrication and Imaging Core Laboratory, King Abdullah University of Science \& Technology (KAUST), \\ Thuwal 23955-6900, Saudi Arabia \\ *Corresponding author: boon.ooi@kaust.edu.sa
}

Received July 23, 2013; revised August 20, 2013; accepted August 22, 2013;

posted August 23, 2013 (Doc. ID 194360); published September 17, 2013

\begin{abstract}
We report on the quantitative evidence of simultaneous amplified spontaneous emission from the AlGaInAs/InAs/ InP-based quantum-well (Qwell) and quantum-dashes (Qdash) in a multistack dash-in-an-asymmetric-well superluminescent diode heterostructure. As a result, an emission bandwidth (full width at half-maximum) of $>700 \mathrm{~nm}$ is achieved, covering entire O-E-S-C-L-U communication bands, and a maximum continuous wave output power of $1.3 \mathrm{~mW}$, from this device structure. This demonstration paves a way to bridge entire telecommunication bands through proper optimization of device gain region, bringing significant advances and impact to a variety of cross-disciplinary field applications. (c) 2013 Optical Society of America

OCIS codes: (160.6000) Semiconductor materials; (250.0250) Optoelectronics; (230.5590) Quantum-well, -wire and -dot devices; (250.5590) Quantum-well, -wire and -dot devices; (230.3670) Light-emitting diodes.

http://dx.doi.org/10.1364/OL.38.003720
\end{abstract}

Ultrabroad and continuous spectrum generation, or supercontinuum generation, is an interesting physical phenomenon and an attractive technology for many applications in optics fiber communications, spectroscopy and sensing, metrology, and imaging $[\underline{1}, 2]$. To date, most commercial ultrabroadband sources are based on nonlinear optical transformations of ultrashort laser pulses and photonic crystal fiber based approaches, which often involve an expensive and bulky high-power laser as pump source, and utilize complex filtering systems [3,4]. For many practical applications, a compact, efficient, and cost-effective broadband device is preferred. In this respect, GaAs- and InP-based semiconductor light emitters have attracted significant attention in the past decade due to their interband transition operation of quantum confined heterostructure at near-infrared region, which facilitated them being a viable platform, inheriting a majority of the above characteristics. Devices in the form of superluminescent diodes (SLDs) [므그 ] and laser diodes [ $\underline{8}-11]$ encompassing the natural large size inhomogeneity of the self-assembled quantum dots (Qdots)/Qdashes have been demonstrated to inherit broad gain spectra, which fundamentally defines the emission bandwidth. Wideband emission from SLDs utilizing a different configuration, for instance, chirping the energy levels of the multistack layers in the active region, single- and multisection device intermixing techniques, has already been reported, with maximum spectral bandwidth in the range of 200-300 nm [7,12]. Recently, a hybrid platform utilizing Qwells-Qdots in the active region of the SLD was proposed and demonstrated with an emission bandwidth $\sim 210 \mathrm{~nm}$ and pulsed output power of $\sim 1.0 \mathrm{~mW}$ [13]. However, the emission range covers only the O-E communication bands.

In this work, we demonstrate the possibility of combining emission from Qwells and Qdashes in the dash-in-a-well active region, of a p-i-n SLD, in realizing an extraordinary emission bandwidth of $>700 \mathrm{~nm}$ at high injections. Through chirping the multistack InAs/InP Qdash structure by varying the AlGaInAs barrier layers thickness, a wavelength coverage of $\sim 1.35-1.75 \mu \mathrm{m}$ (E to $\mathrm{U}$ bands) by Qdashes, and $\sim 1.20-1.35 \mu \mathrm{m}$ (O band) by Qwells, leads to the realization of $>900 \mathrm{~nm}$ spectral width or coverage ( $-30 \mathrm{~dB}$ bandwidth). An output power of $1.3 \mathrm{~mW}$ is demonstrated from this device structure under continuous wave (CW) operation at room temperature. Our results further validate the coexistence of Qwells and Qdashes in a dash-in-a-well heterostructure, and may direct to an entirely different active region platform for realizing ultrabroad emission devices by optimized bandgap engineering.

The chirped device structure (CDS) was grown on a (100) oriented n-type S-doped InP substrate with the active region being a dash-in-an-asymmetric-well structure consisting of four stacks of InAs dashes embedded in Qwells. Each of the five-monolayers-thick InAs dash layers is sandwiched within a $7.6 \mathrm{~nm}$ thick compressively strained $\mathrm{In}_{0.64} \mathrm{Ga}_{0.16} \mathrm{Al}_{0.2} \mathrm{As}$ asymmetric Qwell separated by varying thickness $(20,15,10$, and $10 \mathrm{~nm})$ tensilestrained $\mathrm{In}_{0.50} \mathrm{Ga}_{0.32} \mathrm{Al}_{0.18}$ As top barriers, starting from a $25 \mathrm{~nm}$ thick bottom barrier. More details of the structure can be found from [10]. In addition, a partial four-stack Qwell (7.6 nm thickness) equivalent structure (QWS) was also grown with identical epitaxy to the chirped structure but without the Qdash layers to identify the emission peak. A $50 \mu \mathrm{m}$ oxide-strip broad area SLD was fabricated using the standard laser fabrication process and the partial suppression of Fabry-Perot resonances in the optical path is achieved via the integration of a photon absorber (PA) section to a $1.0 \mathrm{~mm}$ long pump (gain section) section [5,6]. For absorption measurements, $3 \mu \mathrm{m} \times 1000 \mu \mathrm{m}$ ridge-waveguide multisection devices were fabricated with $10 \mu \mathrm{m}$ isolation between each section [14]. The nonfacet-coated SLD devices were tested on a brass heat 
sink using a thermoelectric cooler operated at $20^{\circ} \mathrm{C}$, under $\mathrm{CW}$ operation, by pumping only the gain section while the PA section was left unpumped.

Figure 1(a) shows the amplified spontaneous emission (ASE) output spectrum of our chirped $50 \mu \mathrm{m} \times 1000 \mu \mathrm{m}$ Qdash SLD device under CW operation. Increasing the current injection density $(J)$ resulted in widening of the spectral bandwidth [measured at full width at halfmaximum (FWHM)] and the wavelength coverage. At a drive current density of $J=2.0 \mathrm{kA} / \mathrm{cm}^{2}$, a bandwidth of $>135 \mathrm{~nm}$ is achieved, centered at $\sim 1.61 \mu \mathrm{m}$. The corresponding spectrum ripple over $10 \mathrm{~nm}$ spans from the central wavelength is $<0.2 \mathrm{~dB}$, as shown in the inset of Fig. 1(a). A maximum output power of $>0.35 \mathrm{~mW}$ is achieved at this particular current injection, as illustrated in Fig. 1(b). A visible superlinear L-I curve [left inset of Fig. 1(b)] depicts the transition from the spontaneous emission to the ASE regime. All these characteristics, in addition to no sharp thermal roll-over of the L-I curve, make this device a very attractive broadband emitter. Furthermore, we were able to achieve a maximum power of $\sim 1.3 \mathrm{~mW}$ from $4 \mu \mathrm{m} \times 2500 \mu \mathrm{m}$ SLD [shown in the right inset of Fig. 1(b)] with a single-pass amplification of spontaneous emission, and similar ultrabroad emission spectrum profile but at comparatively larger injection current density values. The increase in the power

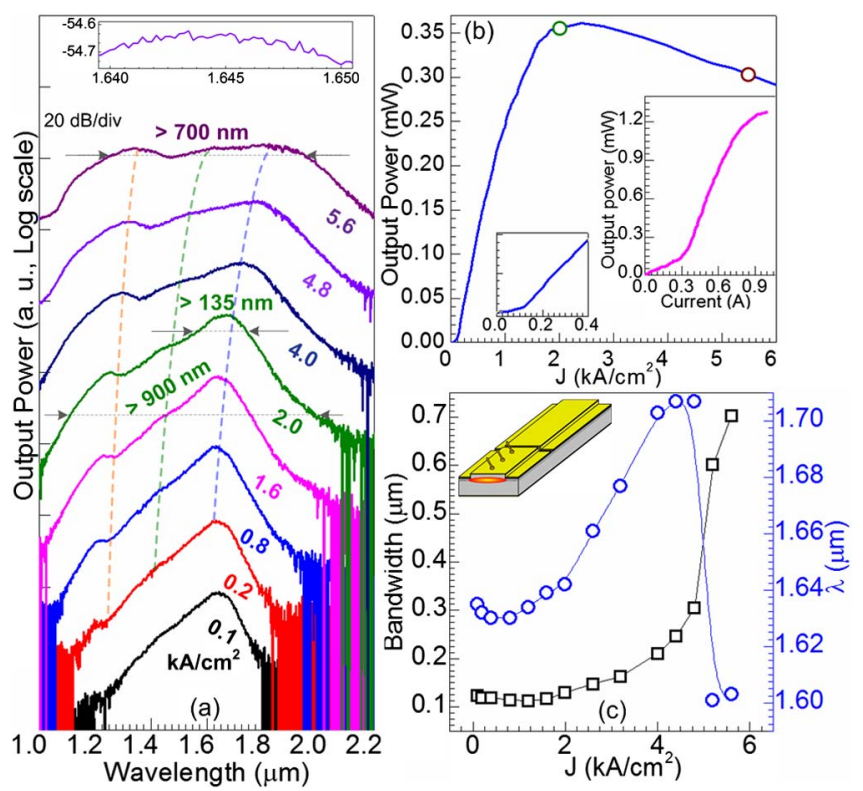

Fig. 1. (a) Room temperature $\mathrm{CW}$ emission spectra of the fabricated $50 \mu \mathrm{m} \times 1000 \mu \mathrm{m}$ chirped Qdash SLD device at different injection current density. The inset shows the spectra ripple within $10 \mathrm{~nm}$ from the central wavelength, at $2.0 \mathrm{kA} / \mathrm{cm}^{2}$. (b) The corresponding L-I characteristics, and (c) change in the emission bandwidth and the central emission wavelength with increasing injection current density. The left inset of (b) shows the zoomed L-I curve at low injection while the right inset shows the L-I characteristics of a $4 \mu \mathrm{m} \times 2500 \mu \mathrm{m}$ SLD device, measured at room temperature under CW operation. A huge emission wavelength coverage of $>900 \mathrm{~nm}$ at $2.0 \mathrm{kA} / \mathrm{cm}^{2}$, and bandwidth exceeding $>700 \mathrm{~nm}$ at $5.6 \mathrm{kA} / \mathrm{cm}^{2}$, is observed [shown as an open symbol in the L-I curve of (b)]. The lines are guide to the eyes showing collective emission from various energy levels of the active region responsible for this observation. value by a factor of four highlights the capability of our bandgap engineered active region. It is noteworthy to mention that these power values correspond to our no-coated single facet results, which could further be increased by various techniques, for instance, facet coatings (antireflection coating), utilizing longer tapered and multisection devices [15], semiconductor optical amplifier in tandem with the SLD devices [16]. The effect of increasing the injection current on the emission bandwidth and the central emission wavelength (calculated by identifying the central wavelength at the FWHM) is shown in Fig. 1(c). We noted an extraordinary broad wavelength coverage of $>900 \mathrm{~nm}$ from our device at $J=2.0 \mathrm{kA} / \mathrm{cm}^{2}$, a direct effect of chirping the active layer which leads to enhanced inhomogeneous broadening. The spectra shows three visible emission humps [marked by dashed lines in Fig. 1(a)] at $~ 1.22, ~ 1.41$, and $\sim 1.61 \mu \mathrm{m}$, dictating the existence of three dominant energy groups in the active region. We attribute these humps to the emissions originating from the Qwells, short- and large-average dash height ensembles, respectively, as illustrated in an energy band model sketch in Fig. 2. We postulate that the 10 and $15 \mathrm{~nm}$ Qdash stacks work collectively under the $\mathrm{CW}$ operation, because of a possible carrier tunneling process among themselves [17], and emit at longer wavelengths because of their large-average dash height (LD), a result of the pronounced effect of vertical strain on the dash formation. The other dash group corresponds to the $20 \mathrm{~nm}$ barrier thickness overgrown Qdash layer with short-average dash height (SD). We have discussed the characteristic of this chirped structure in a greater detail elsewhere [10].

Figure 1(a) depicts that the LD ensemble basically dominates the ASE spectra at low injections $\left(J \leq 0.5 \mathrm{kA} / \mathrm{cm}^{2}\right)$. This could be understood qualitatively by referring to the energy band model at low injection [Fig. 2(a)]. In a Qdash active medium with highly dispersive dash sizes, the emission preferentially occurs in the SD assembly due to their inherent dot-like characteristics viz. small active gain volume, tighter lateral carrier confinement, lower modal gain. However, the $\mathrm{LD}$ ensemble, i.e., larger active volume, has higher modal gain due to large optical power filling factor and small transition energies, and therefore absorbs the high energy photons
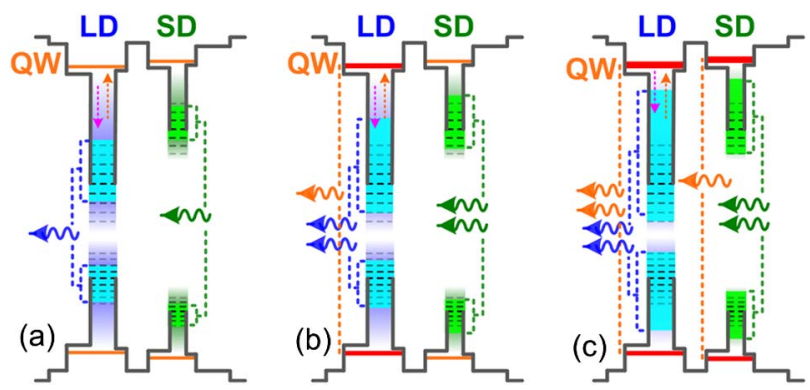

Fig. 2. Energy band model sketch of the chirped Qdash SLD showing the dominant emissions from Qwell (QW), long (LD)and short (SD)-dash assemblies, at (a) low, (b) moderate, and (c) high current injection density. Orange, blue, and olive colors correspond to the available states while red, cyan, and green correspond to the occupied states that are recombining in the system, for the Qwell, long- and short-dash ensembles, respectively. 
from the SD groups thereby dominating the emission operation. This is visible as a single $\sim 1.61 \mu \mathrm{m}$ emission peak at low injection. Note that a small blue shift in the central emission wavelength is observed with increasing injection [Fig. 1(c)]. This is probably caused by competition between the two dash groups with increased collective emission. Another possible reason could be the band filling effect of localized energy states of the dash groups leading to the broadening and blueshifting of the spectra. At moderate injection $\left(0.5 \mathrm{kA} / \mathrm{cm}^{2} \leq J \leq 2.0 \mathrm{kA} / \mathrm{cm}^{2}\right)$, a clear redshift in the central emission wavelength is a signature of the dominating $\mathrm{LD}$ ensemble as more number of dashes start to recombine in the system. This in turn increases the photon reabsorption process, i.e., increased carrier feeding mechanism via optical pumping of the LD group from the SD group. Nevertheless, under this current injection regime, radiative emission from the SD group starts to appear in the spectra (emission hump at $\sim 1.41 \mu \mathrm{m}$ ). Moreover, emission from Qwells are also legible in this regime, as shown in Fig. 1(a), and sketched in Fig. 2(b). This is readily possible if the system is carrier sufficient, i.e., sustaining emissions from all the quantumconfined nanostructures once exceeding the carrier feeding (via optical pumping) process among themselves. More interestingly, at high injections $\left(J>2.0 \mathrm{kA} / \mathrm{cm}^{2}\right)$, the efficiency of Qwells increases whereas the emission from dash ensembles tends to saturate. At $J>5.0 \mathrm{kA} / \mathrm{cm}^{2}$, the emission probably indicates that all the possible optical transitions of both dash ensembles have been saturated and the spectra completely represents entire dash size distribution, in addition to the short wavelength Qwell emission, leading to an astonishingly extremely wide bandwidth of $>600 \mathrm{~nm}$, while maintaining an signal-to-noise ratio of $>20 \mathrm{~dB}$. The emission mechanism in this injection regime is depicted in Fig. 2(c). The realization of the huge emission bandwidth is possible when the quantum efficiencies of all the quantum-confined nanostructures are comparable, in other words, equivalent rate of carrier recombination. In addition, the broadening of the short wavelength emission hump and the redshift of the SD and LD groups emission wavelengths, are partly attributed to the simultaneous emission from different asymmetric Qwells, and the emission from the increasing number of shorter transition energy dashes from both the dash ensembles, respectively, as illustrated in Fig. 2(c). However, in the $\mathrm{CW}$ operation, and at high current injections, the emission mechanism is also influenced by the junction heating that would affect the localized distribution of carriers within the dash stack and among the stacks, probably due to thermionic carrier escape [18]. This thermally activated carrier from dashes could possibly recombine in the Qwells (pumping of Qwells), thus reducing (increasing) the quantum efficiencies of dash assemblies (Qwells). The carriers may also depopulate from the Qwells to the adjacent Qwell before recombining. In general, the thermal effects together with the dash-in-a-well active region is responsible for the observation of $>700 \mathrm{~nm}$ emission bandwidth at $5.6 \mathrm{kA} / \mathrm{cm}^{2}$, with a total output power of $\sim 0.30 \mathrm{~mW}$. Note that a slow power rolloff is observed in our device. Thermal effects are usually undesirable since they degrade the device performance; however, in our case, we capitalize on this effect to pump the Qwells, thus showing its benefit.

In order to confirm our attribution of the emission wavelength humps to different quantum-confined nanostructures in the active region, we carried out the photoluminescence (PL) and absorption measurements. Figure 3(a) plots the PL from the CDS and QWS samples at different excitation power densities, taken at $77 \mathrm{~K}$. In addition, we also show the $77 \mathrm{~K}$ emission spectra of the $\mathrm{SLD}$ at $J=3.0 \mathrm{kA} / \mathrm{cm}^{2}$ in the same figure. The PL peak wavelengths of the CDS and QWS sample at low $\left(1.5 \mathrm{~W} / \mathrm{cm}^{2}\right)$ and high $\left(3.0 \mathrm{~kW} / \mathrm{cm}^{2}\right)$ excitation power densities, agree well with the short (Qwell) and long (LD ensemble) wavelength emission humps, attaining values $\sim 1.17 \mu \mathrm{m}$ and $\sim 1.52 \mu \mathrm{m}$, respectively. The weak emission at $\sim 1.36 \mu \mathrm{m}$ overlaps with the CDS sample's short wavelength PL emission region, at high excitation power density of $3.0 \mathrm{~kW} / \mathrm{cm}^{2}$, further substantiating the emission being contributed by the SD dashes. For additional details on PL analysis, the readers are referred to [10]. In another experiment, we performed the absorption measurements of the CDS sample at room temperature utilizing the multisection ridge-waveguide device and compared with the SLD ASE spectra in Fig. 3(b). Three absorption peaks are apparent from the absorption curve, at high injection. While the two long wavelength peaks persist even at low injection, the short wavelength peak was only visible at high injections $\left(J>5.0 \mathrm{kA} / \mathrm{cm}^{2}\right)$. The peaks agree well with the room temperature emission humps of the SLD ASE spectra, further confirming the role of Qwells and Qdashes (SD and LD assemblies) in achieving an ultrawide emission bandwidth. Note that the peak loss value of the LD groups is far larger than the SD group ( $>$ by $13 \mathrm{~cm}^{-1}$ ) compared to Qwells ( $>$ by $8 \mathrm{~cm}^{-1}$ ), suggesting that the
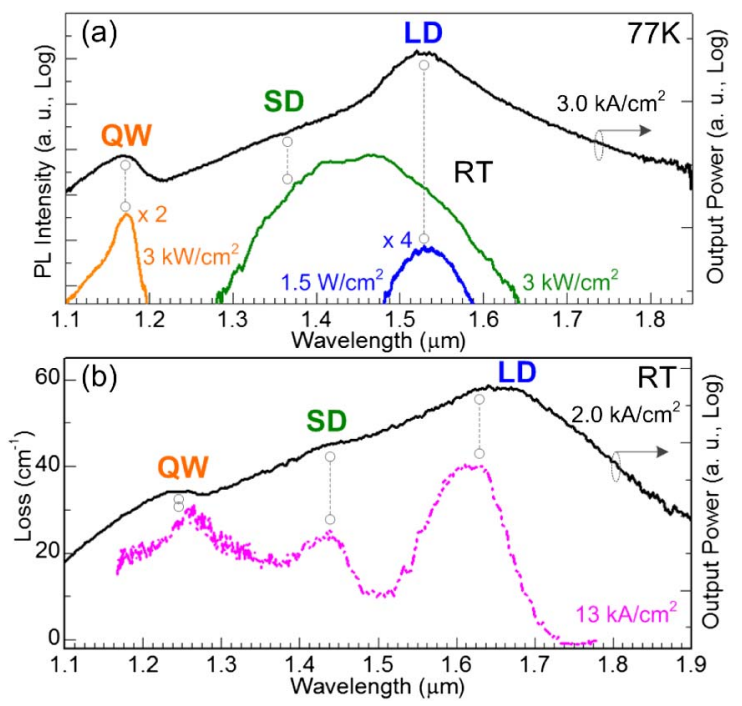

Fig. 3. (a) Comparison of the $77 \mathrm{~K}$ PL spectra of the QWS and CDS samples with the ASE spectrum of the chirped Qdash SLD, at $77 \mathrm{~K}$. The excitation power density value of the PL samples and the injection current density of the SLD are shown in (a). (b) Comparison of the absorption spectra with the ASE spectrum of the chirped Qdash SLD, at room temperature (RT). The injection current densities of the absorption spectra and the Qdash SLD ASE spectrum are shown in (b). 
SD group's gain is probably limited. Moreover, observation and competing absorption peak loss from Qwells suggests that the current feeding via optical pumping mechanism is only possible at high injection currents, which is exactly what has been observed in our SLD analysis. Therefore, our results call for a possible optimization of the gain medium (properly bandgap engineering), perhaps dual $20 \mathrm{~nm}$ barrier thickness dash stacks can be utilized, in addition to an optimized design of the Qwell heights. Through monolithic integration of different bandgap gain sections using multisection device intermixing [19], the emission coverage could further be increased. Moreover, a hybrid Qwell and Qdash structure seems to be an attractive candidate for the same, recently reported with Qdot active medium [13].

In conclusion, we demonstrated the coexistence of Qwells and Qdashes in the dash-in-a-well-chirped InAs/ InP SLD structure. The spectral coverage (emission bandwidth) is extended to $>900 \mathrm{~nm}(>700 \mathrm{~nm})$ due exclusively to the collective emission from the Qwells and Qdashes, thus covering the entire $\mathrm{O}$ to $\mathrm{U}$ communication band. This demonstration shows the feasibility of attaining such a wide emission bandwidth from a compact and single semiconductor device.

The work was supported by KAUST's Competitive Research Grant CRG-1-2012-OOI-010.

\section{References}

1. C. Gmachl, D. L. Sivco, R. Colombelli, F. Capasso, and A. Y. Cho, Nature 415, 883 (2002).

2. B. S. Ooi, H. S. Djie, Y. Wang, C. L. Tan, J. C. M. Hwang, X. M. Fang, J. M. Fastenau, A. W. K. Liu, G. T. Dang, and W. H. Chang, IEEE J. Sel. Top. Quantum Electron. 14, 1230 (2008).

3. A. Unterhuber, B. Považay, K. Bizheva, B. Hermann, H. Sattmann, A. Stingl, T. Le, M. Seefeld, R. Menzel, and M. Preusser, Phys. Med. Biol. 49, 1235 (2004).
4. W. J. Wadsworth, A. Ortigosa-Blanch, J. C. Knight, T. A. Birks, T.-P. M. Man, and P. S. J. Russell, J. Opt. Soc. Am. B 19, 2148 (2002).

5. H. S. Djie, C. E. Dimas, and B. S. Ooi, IEEE Photon. Technol. Lett. 18, 1747 (2006).

6. H. S. Djie, C. E. Dimas, D.-N. Wang, B.-S. Ooi, J. C. Hwang, G. T. Dang, and W. H. Chang, IEEE Sens. J. 7, 251 (2007).

7. Z. Y. Zhang, R. A. Hogg, X. Q. Lv, and Z. G. Wang, Adv. Opt. Photon. 2, 201 (2010).

8. H. S. Djie, B. S. Ooi, X. M. Fang, Y. Wu, J. M. Fastenau, W. Liu, and M. Hopkinson, Opt. Lett. 32, 44 (2007).

9. H. S. Djie, C. L. Tan, B. S. Ooi, J. C. M. Hwang, X. M. Fang, Y. Wu, J. M. Fastenau, W. K. Liu, G. T. Dang, and W. H. Chang, Appl. Phys. Lett. 91, 111116 (2007).

10. M. Z. M. Khan, T. K. Ng, C.-S. Lee, P. Bhattacharya, and B. S. Ooi, Appl. Phys. Lett. 102, 091102 (2013).

11. M. Z. M. Khan, T. K. Ng, U. Schwingenschlogl, and B. S. Ooi, IEEE J. Quantum Electron. 48, 608 (2012).

12. S. Haffouz, P. Barrios, R. Normandin, D. Poitras, and Z. Lu, Opt. Lett. 37, 1103 (2012).

13. S. Chen, K. Zhou, Z. Zhang, J. Orahcrd, D. Childs, M. Hugues, O. Wada, and R. Hogg, IEEE J. Sel. Top. Quantum Electron. 19, 1900209 (2013).

14. Y.-C. Xin, Y. Li, A. Martinez, T. J. Rotter, H. Su, L. Zhang, A. L. Gray, S. Luong, K. Sun, Z. Zou, J. Zilko, P. M. Varangis, and L. F. Lester, IEEE J. Quantum Electron. 42, 725 (2006).

15. M. A. Majid, M. Hugues, S. Vezian, and R. Hogg, IEEE Photon. J. 4, 2066 (2012).

16. X. Li, P. Jin, Q. An, Z. Wang, X. Lv, H. Wei, J. Wu, J. Wu, and Z. Wang, Nanoscale Res. Lett. 6, 1 (2011).

17. P. Miska, J. Even, C. Paranthoen, O. Dehaese, A. Jbeli, M. Senes, and X. Marie, Appl. Phys. Lett. 86, 111905 (2005).

18. C. L. Tan, H. S. Djie, Y. Wang, C. E. Dimas, V. Hongpinyo, Y. H. Ding, and B. S. Ooi, IEEE Photon. Technol. Lett. 21, 30 (2009).

19. B. S. Ooi, K. McIlvaney, M. W. Street, A. S. Helmy, S. G. Ayling, A. C. Bryce, J. H. Marsh, and J. Roberts, IEEE J. Quantum Electron. 33, 1784 (1997). 